\title{
Light- and temperature-entrained circadian regulation of activity and mRNA accumulation of 1-aminocyclopropane-1-carboxylic acid oxidase in Stellaria longipes
}

\author{
Arumugam Kathiresan, D.M. Reid, C.C. Chinnappa \\ Department of Biological Sciences, The University of Calgary, Calgary, Alberta, Canada T2N 1N4 \\ Planta (1996) 199:329-335
}

In the above article the authors forgot to cite the Genbank accession number and the designer of the primers. This information is provided below.

The cDNA fragment encoding $\mathrm{ACC}$ oxidase from the cDNA library was isolated by using the oligonucleotide primers homologous to conserved regions of ACC oxidase cDNAs of sunflower designed by Dr. J.-H. Liu, Department of Biological Sciences, The University of Calgary, Calgary, AB, Canada (Genbank accession no. L21976), tomato (X58885), and petunia (L212976). 